\title{
A mechanical impact of coatings on membranes
}

\author{
Hansen, Michael Søren; Reus, Roger De; Eriksen, Gert Friis; Bouwstra, Siebe
}

Published in:

Proceedings on The 14th IEEE International Conference on Micro Electro Mechanical Systems

Link to article, DOI:

10.1109/MEMSYS.2001.906553

Publication date:

2001

Document Version

Publisher's PDF, also known as Version of record

Link back to DTU Orbit

Citation (APA):

Hansen, M. S., Reus, R. D., Eriksen, G. F., \& Bouwstra, S. (2001). A mechanical impact of coatings on membranes. In Proceedings on The 14th IEEE International Conference on Micro Electro Mechanical Systems (pp. 361-364) https://doi.org/10.1109/MEMSYS.2001.906553

\section{General rights}

Copyright and moral rights for the publications made accessible in the public portal are retained by the authors and/or other copyright owners and it is a condition of accessing publications that users recognise and abide by the legal requirements associated with these rights.

- Users may download and print one copy of any publication from the public portal for the purpose of private study or research.

- You may not further distribute the material or use it for any profit-making activity or commercial gain

- You may freely distribute the URL identifying the publication in the public portal 


\title{
A MECHANICAL IMPACT OF COATINGS ON MEMBRANES
}

\author{
M. S. Hansen ${ }^{1}$, R. de Reus ${ }^{1}$, G. F. Eriksen ${ }^{2}$ and S. Bouwstra ${ }^{1}$ \\ ${ }^{1}$ Mikroelektronik Centret, DTU, bldg. 345 East, DK-2800 Lyngby, Denmark \\ ${ }^{2}$ Grundfos A/S, Denmark \\ e-mail: mha@mic.dtu.dk
}

\begin{abstract}
The mechanical impact of coatings containing residual stress on membranes is investigated. Closed-form formulas describing this impact are presented and verified using both finite elements modeling and physical experiments. Theory and experiments are in good agreement. Thus, a simple tool for design of coated pressure sensors is provided.
\end{abstract}

\section{INTRODUCTION}

For the use of differential pressure sensors in aggressive media protection of the membrane by a thin film protective coating has been suggested [1].For true differential pressure sensors, a double-sided coating is required. Residual stresses are likely to occur in such coatings. The mechanical impact of coatings on membranes is important to know in order to predict the sensitivity of the pressure sensor.

The influence of residual stress on uncoated membranes on the sensitivity of membranes has previously been investigated and presented in [2]. Changes in the deformation, strain and deflection, at respectively the centre and the edges of the membrane can be calculated from Eq. (1) and (2), as a function of the residual strain, $\epsilon_{\text {res }}$, normalized with the slimness squared, $(a / t)^{2}$ :

$$
\begin{aligned}
f_{\text {center }}\left(\epsilon_{\text {res }}(a / t)^{2}\right) & =\frac{f_{\text {center }}\left(\epsilon_{\text {res }}=0\right)}{1+\alpha \times \epsilon_{\text {res }}(a / t)^{2}} \\
f_{\text {edge }}\left(\epsilon_{\text {res }}(a / t)^{2}\right) & =\frac{f_{\text {edge }}\left(\epsilon_{\text {res }}=0\right)}{\sqrt{1+\alpha^{\prime} \times \epsilon_{\text {res }}(a / t)^{2}}}
\end{aligned} .
$$

in which $\alpha$ is a constant. For values of $\alpha$ see Table. 1 .

A theory describing the changed stiffness of the layered structure as compared to the uncoated membrane has been suggested in [3]:

$$
\begin{gathered}
f_{\text {center }}\left(\epsilon_{\text {res }, \text { coat }}(a / t)^{2}\right)_{\text {coated }}= \\
\frac{f_{\text {center }}\left(\epsilon_{\text {res }, \text { coat }}(a / t)^{2}=0\right)_{\text {uncoated }}}{\Delta+\epsilon_{\text {res }, \text { coat }}(a / t)^{2} 2 \alpha \chi \xi} \\
f_{\text {edge }}\left(\epsilon_{\text {res,coat }}(a / t)^{2}\right)_{\text {coated }}= \\
\frac{f_{\text {edge }}\left(\epsilon_{\text {res }, \text { coat }}(a / t)^{2}=0\right)_{\text {uncoated }}}{\Delta \sqrt{1+\epsilon_{\text {res }, \text { coat }}(a / t)^{2} 2 \alpha^{\prime} \frac{2 \xi \chi}{\Delta}}}
\end{gathered}
$$

Table 1: Values of $\alpha$ for deflection and bending strain.

\begin{tabular}{ccc}
\hline \hline location & deflection & bending strain \\
\hline center & 0.290 & 0.445 \\
mid-edge & - & 0.391 \\
\hline \hline with $\quad \Delta=1+2 \times \chi\left((1+2 \times \xi)^{3}-1\right)$ \\
here $\quad \chi=\frac{E_{\text {coat }}}{E_{\text {memb }}} \frac{\left(1-\nu_{\text {memb }}^{2}\right)}{\left(1-\nu_{\text {coat }}^{2}\right)}$ and $\xi=\frac{t_{\text {coat }}}{t_{\text {memb }}}$
\end{tabular}

$\Delta$ denotes the impact of a coating of zero residual stress on a membrane of zero residual stress. $\chi$ represents the coating's material stiffness, which depends on Young's modulus, $E$, and Poisson's ratio, $\nu$, relative to the membrane's material stiffness. $\xi$ is the ratio between coating and membrane thickness. $\epsilon_{\text {res,coat }}(a / t)^{2}$ is the residual stress in the coating normalized to the slimness of the membrane squared.

Physical experiments and finite element modeling, FEM, using ANSYS were carried out to verify this suggested theory. Only square membranes were investigated.

A new design of differential pressure sensors, which avoids step-coverage problems of the coating, uses a deposited membrane on top of the piezoresistors and interconnects [5]. In this design the piezoresistors are located at the backside of the membrane (see Fig. 1b).

Whether the piezoresistors are located at the front- or at the backside of the membrane changes the strain profile in the region of piezoresistors, due to the change of local geometry from a flat surface (see Fig 1a) to a relatively sharp edge (see Fig 1b).

A change in the strain level, due to a different location, will be shown as a change of the value of the constant in Eq. (5), which shows the relation between the strain and the applied pressure, $q$ :

$$
\epsilon=\text { const } \times \frac{q a^{2}\left(1-\nu^{2}\right)}{E t^{2}}
$$

Pressure sensors are normally only subjected to minor deformation in order to maintain linear deformation, which then can be predicted by using Eq. (5). In order to get the largest possible operation-range of a pressure sensor it is important to know when the non-linear deformations have a major influence. Therefore the maximum level of relative 
(a)

(b)

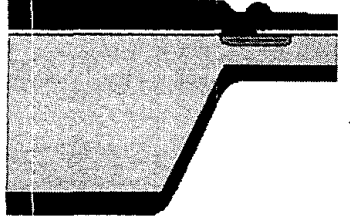

Piezoresistor on top of membrane

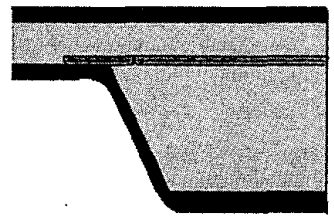

Membrane on top of piezoresistor (deposited membrane)
Figure 1: Cross-section of $\mathrm{KOH}$-etched silicon membrane. The piezoresistors can either be placed on the front- (a) or on the backside (b) of the membrane. Interconnects were not included in the simulations.

deformations was determined for two different criteria: When the non-linear deformation is respectively $1 \%$ and $10 \%$ of the total deformation.

\section{FEM EXPERIMENTS}

Finite element modeling of layered structures can either be done using normal solid elements and individual modeling of each layer or by using layered elements (solid or shell), where one element can contain multiple layers. No significant differences between these two approaches were observed.

Very good accordance between results derived using ANSYS and theory is obtained for centre deflection, within $2 \%$. For bending strain at the centre and at the middle of the edges of the membrane, see Fig. 2, a good accordance is found for tensile normalized residual strains, $\epsilon_{\text {res,coat }}(a / t)^{2}$, and minor compressive normalized residual strain. The range of the normalized residual strain is from -10 to 10 for most practical applications of thin films as protective

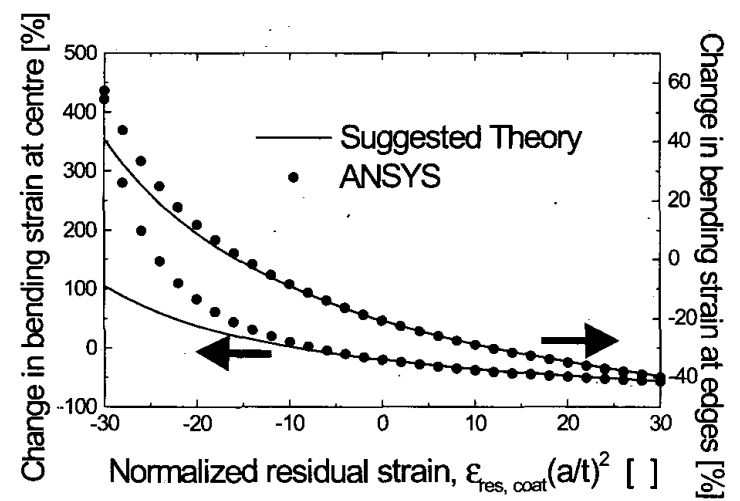

Figure 2: Change in strain at centre and at the middle of the edges of the membrane. $\xi=1 / 25$. coating on membranes in pressure sensors. Within this range the accordance between the theory and the results derived using FEM is very good: within $5 \%$ for the entire range and mostly within $1 \%$.

Finite element modeling has been carried out in order to measure the difference of the strain level at the front- or the backside of the membrane, when the membrane is produced using a $\mathrm{KOH}$-etch (see Fig. 1). Both these scenarios have been compared to the ideally clamped boundary condition neglecting the sloping sidewalls. For these experiments the solid45 element in ANSYS has been used. Interconnects were not included in the simulations, and the strain levels were calculated for the outer layers of the membrane, neglecting that the piezoresistors do have a thickneiss.

Besides the expected difference of the strain-level due to changes of the location from the front- to the backside of the membrane, the dependence on the slimness has also been determined, see Fig 3 .

\section{PHYSICAL EXPERIMENTS}

The mechanical impact of coatings on membranes was determined physically by applying a differential pressure over a silicon wafer containing $\mathrm{KOH}$-etched membranes. The deflection profiles of the membranes were measured optically using a UBM autofocus system with a vertical resolution of $0.03 \mu \mathrm{m}$ and horizontal resolution of $1 \mu \mathrm{m}$. The same membrane was measured, before a coating was deposited, with coating deposited and after removal of the coating. The bending strain along the deflection profile can be derived from the deflection profile: $\epsilon_{\text {tend }}=-t / 2 \times \frac{\partial^{2} w}{\partial x^{2}}$ according to the general theory of linear deflection found in [4]. This was also done, see Fig. 4. Center deflection and bending strain, both at the center and at the edges, were determined from the measured surfaceprofile. Considerable inaccuracy was present when deriving the bending strain.

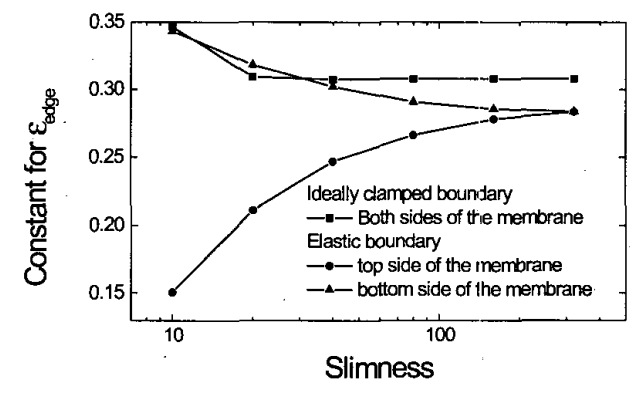

Figure 3: Value of the linear constant for $\epsilon_{\text {edge }}$ as a function of the slimness, for 3 different scenarios: Ideally clamped boundary (squares), elastic boundary at the frontside (circles) and at the backside (triangles) of the membrane. 


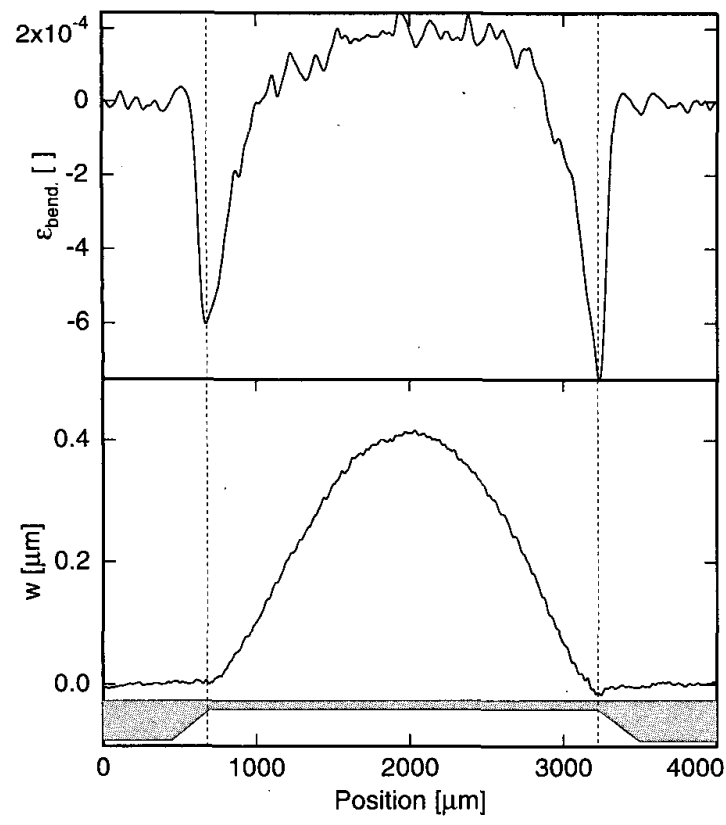

Figure 4: Deflection profile of membrane. Derivation of bending strain from second derivative of the deflection profile.

Amorphous tantalum oxide, $\mathrm{a}-\mathrm{TaO}$ was applied in the above experiment. a-TaO has excellent coating properties [3]. $\chi=1$ for $\mathrm{a}-\mathrm{TaO}$ on silicon. The coatings were sputtered on either side of the membrane and had a thickness of 0.95 $\mu \mathrm{m}$. Measurements were carried out at 4 different residual stress levels, obtained by post-annealing of the coating at different temperatures [3].

\section{RESULTS}

In Table 2 the impacts, both experimental and theoretical, of the coatings can be observed for two membranes, with sidelengths of 1480 and $2620 \mu \mathrm{m}$ and thicknesses of 19.0 and $21.5 \mu m$, respectively. In general the experimental impacts, derived using the more reliable center deflection, are smaller than the theoretical. The residual stress levels were measured using a separate test-wafer and variations of the residual stress levels as-deposited occur. This might be the reason for this general discrepancy between the experimental and theoretical impacts.

The inaccuracy of the physical experiments was determined prior to these experiments and is presented by the error bars in the diagram in Table 2. Including the inaccuracy there is in general accordance with the theoretically and the experimentally derived impacts.

Along the investigation of the impact of different locations of the piezoresistor a dependence of the slimness on the linear constant was discovered, which may be caused by the gradual change of the membrane from a thick plate to a thin plate, which is described by different theories.
Table 2: Impacts of coatings derived theoretically and experimentally.

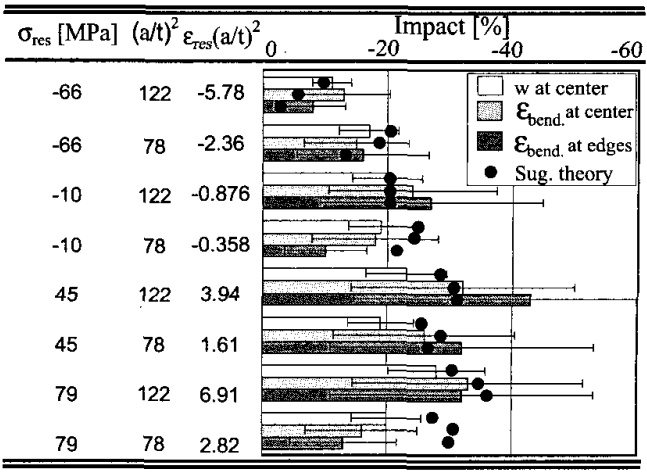

A difference of the strain level at the front- and backside of the membrane is observed and illustrated in Fig 3 as a different value of the constant in Eqn (5). For larger slimnesses the strain-levels converge, but for moderate slimnesses of around 100 there is still a difference of approximately $10 \%$.

Furthermore it can be seen that the strain level at the backside of the membrane as a function of the slimness is similar to the one for the ideally clamped boundary. This is not the case for the strain level at the frontside of the membrane.

The influence of the non-linear behaviour can be seen in Tab 3. For the same degree of non-linearity it requires a different degree of relative deformation when comparing $w_{\text {cent }}, \epsilon_{\text {cent }}$ and $\epsilon_{\text {edge }}$. If piezoresistive read-out is used to measure the pressure, the non-linearity of the strain at the centre and at the edges are of interest. Comparing these two and using a $1 \%$ non-linearity criteria the difference of deformation allowed to fulfill this criteria is a factor of 6 .

\section{CONCLUSIONS}

Closed-form formulas for the impact of coatings on membrane have been presented and have been verified using finite elements modeling and physical experiments.

Using finite element modeling it has been shown that there is a difference in strain level, which the piezoresistors measure, depending on positioned at the front- or the backside of the membrane.

Also, using finite element modeling non-linear deflection has been investigated. Maximum levels of relative deforma-

Table 3: Beginning of non-linear behaviour for $w_{c e n t}, \epsilon_{c e n t}$ and $\epsilon_{e d g e}$. Non-linearity is defined as: $100 \% \times \frac{x_{\text {non-linear }}}{x_{\text {totel }}}$

\begin{tabular}{ccc}
\hline \hline Parameter & $1 \%$ non-linearity & $10 \%$ nonlinearity \\
\hline$w_{\text {cent }}$ & $0.15 \frac{w}{t}$ & $0.45 \frac{w}{t}$ \\
$\epsilon_{\text {cent }}$ & $0.025 \frac{w}{t}$ & $0.2 \frac{w}{t}$ \\
$\epsilon_{\text {edge }}$ & $0.15 \frac{w}{t}$ & $1.0 \frac{w}{t}$ \\
\hline \hline
\end{tabular}


tion were determined for $1 \%$ and $10 \%$ non-linearity.

\section{REFERENCES}

1. K. Dyrbye, T. R. Brown, G. F. Eriksen, J. Micromech. Microeng. 6 (1996) 187-192.

2. J. Bastue and S. Bouwstra Proc. MME'95, Copenhagen, Denmark (1995) 234-238.

3. C. Christensen, Ph.D. Thesis MIC, DTU, Denmark, Dec., 1998 and C. Christensen, R. de Reus and S. Bouwstra

4. S. Timoshenko et al. "Theory of plates and shells" McGraw-Hill, New York, USA (1959)

5. S. Bouaidat, G. F. Eriksen, R. de Reus, P. E. Andersen and S. Bouwstra. Proc. Eurosensors '00., Copenhagen, Denmark, 2000 